\title{
Case report: primary thyroid lymphoma presenting as a rapidly enlarging thyroid mass
}

\begin{abstract}
Primary thyroid lymphoma is a rarely encountered clinical entity that occurs mainly in elderly females. We present a case of B-cell origin thyroid lymphoma. The diagnosis was made by combined histology and immunochemistry. A 79-year-old woman presented with an enlarging neck mass with compression signs, dysphagia and pressure sensation around the neck. On admission, the sonogram of the thyroid gland showed an enlarged mass and CT scan demonstrated diffuse enlargement of the thyroid extending on the anterior chest wall. After total thyroidectomy, the histological investigation revealed the presence of a diffuse large B-cell non-Hodgkin's lymphoma without other loci from the systemic staging. The patient underwent chemotherapy and radiation therapy. Clinicians should include primary thyroid lymphoma in the differential diagnosis of a rapidly enlarging thyroid mass.
\end{abstract}

Keywords: primary thyroid lymphoma, non-hodgkin lymphoma, thyroid cancer
Volume I Issue I - 2014

\author{
G Papadakis,' A Tertipi,' M Papazian, ${ }^{2} \mathrm{~K}$ \\ Moustakas,' A Pappas' \\ 'Department of Endocrinology and Diabetes Center, Metaxa \\ Anticancer Hospital, Greece \\ ${ }^{2}$ Department of Pathology, Metaxa Anticancer Hospital, Greece
}

Correspondence: Georgios Papadakis, Department of Endocrinology and Diabetes Center, Metaxa Anticancer Hospital, Mpotasi 5I, Pireaus 18537, Athens, Greece, Tel 00306932598392, Email george.papadakis.md@gmail.com

Received: October 21, 2014 | Published: November 15, 2014
Abbreviations: PTL, primary thyroid lymphomas; MALT, mucosa associated lymphoid tissue

\section{Introduction}

Primary thyroid lymphomas (PTL) are very rare and account for only $2.5 \%$ to $5 \%$ of all thyroid malignancies and $1-2 \%$ of all extranodal malignancies. ${ }^{1}$ PTL is more prevalent in females in the sixth to seventh decade of life. ${ }^{2}$ The presentation of PTL can be puzzling. PLT may be mistaken for anaplastic thyroid cancer. Immunochemistry is an important part of pathologic differentiation of these two entities.

We present here the clinical, morphological and histological features of a PTL which developed in a female patient as a rapidly enlarging thyroid mass. Finally, relevant diagnostic dilemmas are summarized.

\section{Case presentation}

A 79-year-old woman presented to our department reporting a recent-onset gross neck mass, throat discomfort, dysphagia, hoarseness and shortness of breath. She reported no personal or family history of thyroid disease and the symptoms had been noticed 3 months before presentation. The patient had no fever, night sweats or weight loss. The patient was nonsmoker with a remote history of cholecystectomy. The patient had no previous neck radiation exposure. The remaining medical and family history was unremarkable. Hormonal evaluation three months before the admission revealed increased thyrotropin

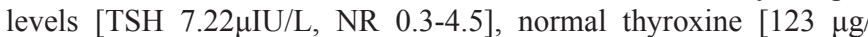
$\mathrm{dl}$, NR 66-181] and normal triiodothyronine levels $[1.81 \mathrm{nmol} / \mathrm{L}$, NR 1.1-3.1]. The antithyroglobulin antibodies were increased (anti$\mathrm{Tg} 109 \mathrm{IU} / \mathrm{ml}, \mathrm{NR}<60)$ and antithyroid peroxidase antibodies were normal (anti-TPO $45 \mathrm{IU} / \mathrm{ml}, \mathrm{NR}<60$ ). The initial sonogram indicated multinodular goiter and a gross heterogeneous hypoechogenic mass on the left lobe (maximum diameter $38 \mathrm{~mm}$ ) compatible with lymphocytic thyroiditis. FNAB was not suggested and the patient was prescribed L-thyroxine $50 \mu \mathrm{g}$ replacement therapy daily.
On admission three months later, the symptoms deteriorated. Clinical examination revealed a firm, immobile and painless mass descending in the anterior chest wall. The laryngoscopy revealed left vocal cord palsy. Biochemical investigation was normal. A new sonogram was performed which revealed a $21 \times 26 \mathrm{~mm}$ solid mass on the right lobe and a $26 \times 29 \mathrm{~mm}$ solid mass on the left lobe. The masses were strongly hypoechoic, heterogeneous intermingled with echogenic structures. These features are related to thyroid lymphoma. ${ }^{3}$ Enlarged neck nodes were observed on both sides. The patient underwent a fine needle aspiration biopsy with a $23 \mathrm{G}$ needle and the cytologic analysis showed thyrocytes, lymphoid elements and atypical nuclei. The histopathologic evaluation for CD-45 was negative (nearly all large B-cell lymphomas are CD-45 positive).

The patient underwent imaging of the neck for further evaluation. CT demonstrated marked enlargement of the thyroid gland, left greater than right. There was mass effect on the trachea, decreased diameter of the trachea and enlarged neck nodes (Figure 1). The patient underwent total thyroidectomy.

Combined histological and immune histological analyses led to the diagnosis of la diffuse large B-cell lymphoma of the follicular centre/ activated like-cell-non GCC, with a high proliferation index (Mib1/Ki-67 : 90\%), positive LCA(CD45Rb), CD10, CD75, CD79a, BSAP, BCL6, BCL2, MUM1, k-light chain, p53 and p63 and negative reaction of the neoplastic cells for CKAE1-3, EMA, HBME1,Thyreogloboulin, Galectin-3 and others. ${ }^{4}$

Evaluation for systemic disease with thorax, abdomen and pelvis imaging and bone marrow aspiration excluded other neoplastic foci. According to the Ann Arbor guidelines for primary thyroid lymphoma the patient had stage IIE disease at diagnosis (involvement of the thyroid and lymph nodes on the same side of the diaphragm). After surgery the patient underwent systemic chemotherapy with the R-CNOP chemotherapeutic protocol, i.e. rituximab (anti CD-20 antibody) $580 \mathrm{mg}$, mitoxandrone $15 \mathrm{mg}$, cyclophosphamide 1160 $\mathrm{mg}$, vincristine $2 \mathrm{mg}$ and methylprednisolone $16 \mathrm{mg}$ cumulative dose. 
Radiation therapy was planned after the chemotherapy cycles. The post-chemotherapy CT scan showed a marked regression of the mass with decompression of the trachea (Figure 3).

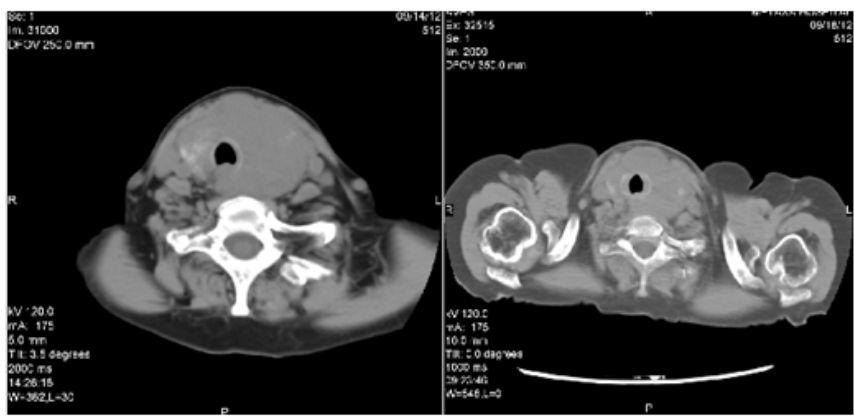

Figure I Neck and thorax CT scan. The pre-treatment figures show a large mass compressing the trachea extending on the anterior chest wall.

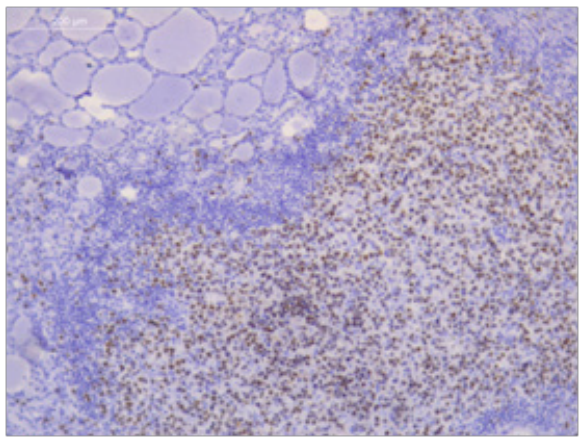

Figure 2A BCL-6 $\times 10$

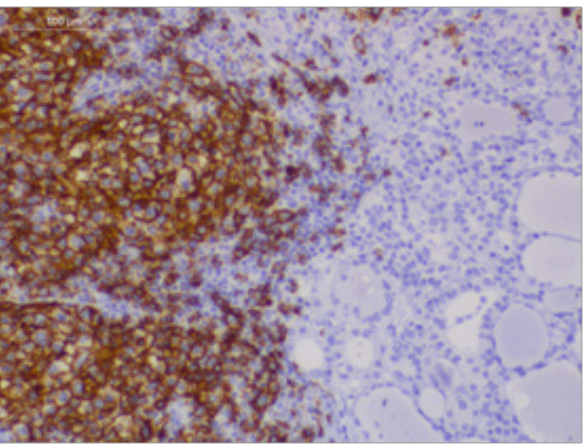

Figure 2B CD20X20

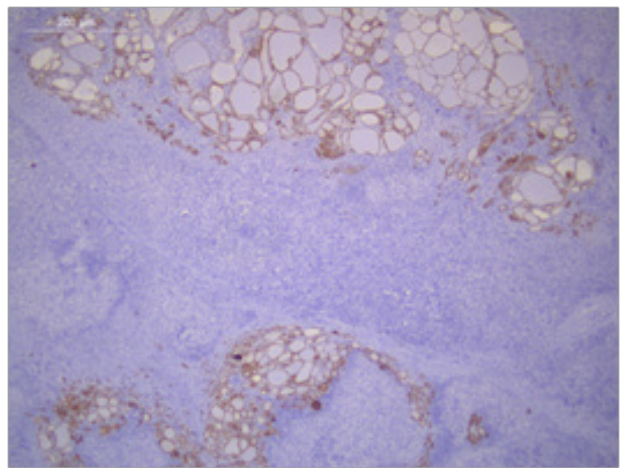

Figure 2C CKAEI-3XI0

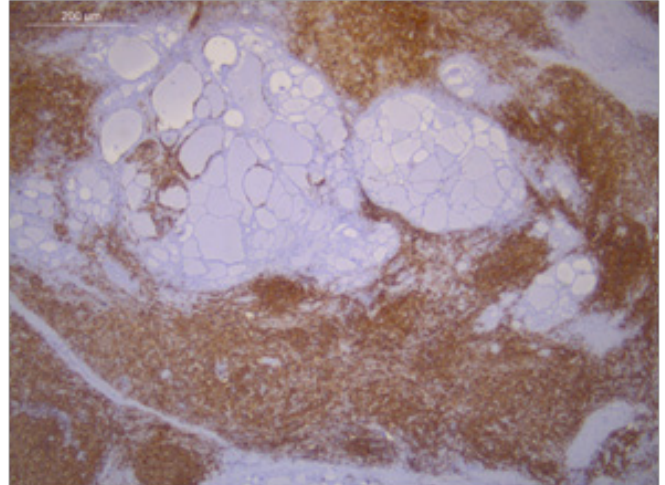

Figure 2D CD20X2,5

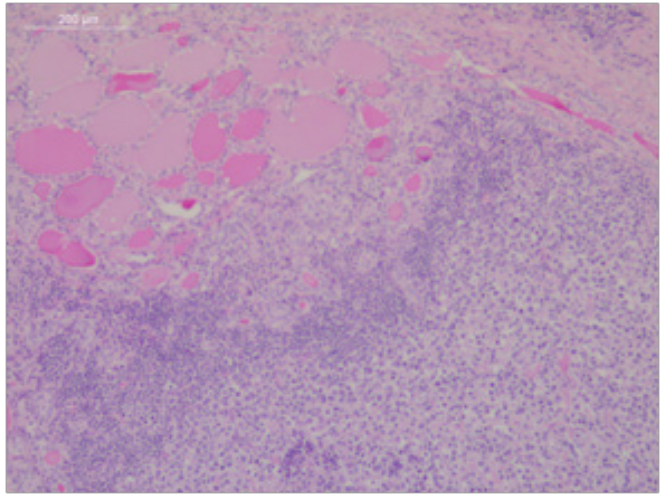

Figure 2E H/E stain

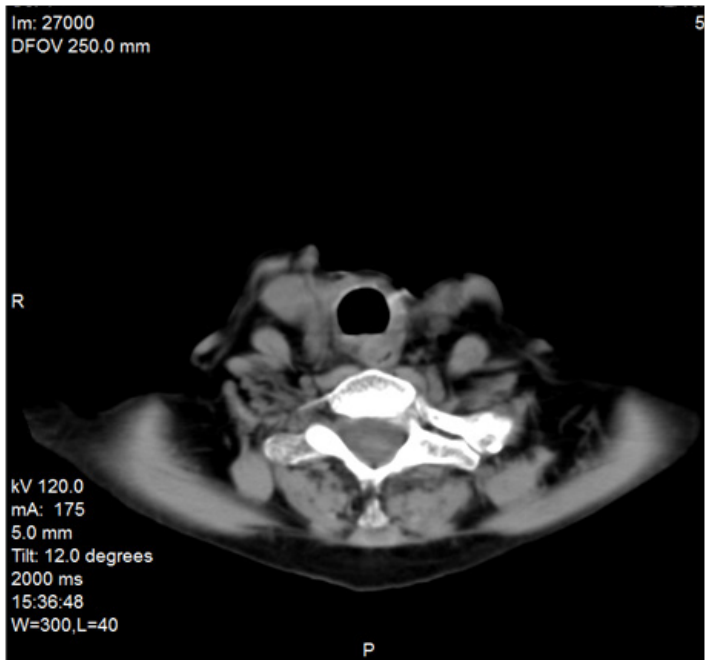

Figure 3 Neck CT-scan after chemotherapy.

\section{Discussion}

We describe an unusual case of a PTL. Within the population, this disease is estimated to occur at an annual incidence rate of 2.1 persons per million. PTL is associated with a poor prognosis. ${ }^{5}$ Most thyroid lymphomas are of B-cell origin. Mucosa associated lymphoid tissue (MALT) is recognized as a distinct lymphoma with unique clinopathological features and follicular lymphoma is another very rare subtype. 
Chronic lymphocytic thyroiditis or Hashimoto's disease is found in more than $90 \%$ of the reported cases. ${ }^{6}$ Some authors hypothesize that chronic stimulation of lymphocytes by thyroiditis leads to malignant transformation. In our patient antithyroglobulin antibodies were increased in multiple blood tests. Most patients with PTL are euthyroid at initial presentation and $10 \%$ present with hypothyroidism. ${ }^{7}$

On presentation, the clinical findings of our patient orientated us to consider anaplastic carcinoma of the thyroid. Most patients with PTL have a rapidly growing painless thyroid mass causing compressive symptoms. These symptoms overlap with that of the anaplastic thyroid carcinoma. ${ }^{8}$ The unexpected histological examination changed the initial diagnosis. The diagnosis of a primary thyroid B-cell lymphoma was established after exclusion of systemic involvement.

FNA is an essential tool in the diagnostic workup of thyroid disease, ${ }^{9}$ although FNA yields inconsistent results in the diagnosis of thyroid lymphomas and its diagnostic accuracy varies widely. This is due to the histopathological similarities between primary thyroid lymphoma and Hashimoto's thyroiditis. ${ }^{10}$ The gold standard for histologic diagnosis is considered the core needle biopsy or surgical biopsy. In our patient the FNAB was non-diagnostic and the cell immunohistochemistry for CD-45 was not positive to establish the diagnosis. FNAB should not replace open thyroid biopsy which is important for the confirmation and sub typing of the disease. Currently, a larger percentage of patients undergo surgery in order to achieve precise histological diagnosis. ${ }^{11}$ In our patient thyroidectomy was useful for the management of compressive symptoms and for the histological diagnosis.

Chemotherapy followed by radiotherapy is considered the standard treatment. ${ }^{12}$ Surgery that was once the mainstay of treatment for PTL plays now a minimal role. Total thyroidectomy exposes unnecessarily the patient to the risks of surgery, such as recurrent laryngeal nerve damage and hyperparathyroidism without conferring any survival advantage. Thyroidectomy is useful for the management of compressive symptoms of the trachea and the esophagus and severe airway obstruction. Logo regional treatment with surgery is the primary treatment of localized MALT lymphomas.

In conclusion, we describe a very rare case of primary thyroid lymphoma. Our report emphasizes the need for clinical awareness in such perplexing cases which clearly require a multidisciplinary approach.

\section{Acknowledgments}

None.

\section{Conflict of interest}

The authors have nothing to disclose, and there is no financial interest or any conflict of interest.

\section{References}

1. Mack LA, Pasieka JL. An evidence-based approach to the treatment of thyroid lymphoma. World J Surg. 2007;31(5):978-986.

2. Graff-Baker A, Roman SA, Thomas DC, et al. Prognosis of primary thyroid lymphoma: demographic, clinical, and pathologic predictors of survival in 1,408 cases. Surgery. 2009;146(6):1105-1115.

3. Nam M, Shin JH, Han BK, et al. Thyroid lymphoma: correlation of radiologic and pathologic features. J Ultrasound Med. 2012;31(4):589594.

4. Katna R, Shet T, Sengar M, et al. Clinicopathologic study and outcome analysis of thyroid lymphomas: Experience from a tertiary cancer center. Head Neck. 2012;35(2):165-171.

5. Antonino A, Rosato A, Barbato F, et al. Thyroid lymphoma: diagnostic pitfalls on pre-operative ago-biopsy. Ann Ital Chir. 2013;84(5):541-544.

6. Watanabe N, Noh JY, Narimatsu H, et al. Clinicopathological features of 171 cases of primary thyroid lymphoma: a long-term study involving 24553 patients with Hashimoto's disease. Br J Haematol. 2011;153(2):236-243.

7. Widder S, Pasieka JL. Primary thyroid lymphomas. Curr Treat Options Oncol. 2004;5(4):307-313.

8. Green LD, Mack L, Pasieka JL. Anaplastic thyroid cancer and primary thyroid lymphoma: a review of these rare thyroid malignancies. J Surg Oncol. 2006;94(8):725-736.

9. Kwak JY, Kim EK, Ko KH, et al. Primary thyroid lymphoma: role of ultrasound-guided needle biopsy. J Ultrasound Med. 2007;26(12):17611765 .

10. Beasley MJ. Lymphoma of the Thyroid and Head and Neck. Clin Oncol. 2012;24(5):345-351.

11. Alzouebi M, Goepel JR, Horsman JM, et al. Primary thyroid lymphoma: the 40 year experience of a UK lymphoma treatment centre. Int J Oncol. 2012;40(6):2075-2080.

12. Ragusa M, Cirocchi R, Puxeddu E, et al. Surgical treatment of primitive thyroid lymphoma. Tumori. 2009;95(6):712-719. 